\title{
Line-by-line opacity stellar model atmospheres
}

\author{
D. Shulyak ${ }^{1,2}$, V. Tsymbal ${ }^{1,2}$, T. Ryabchikova ${ }^{2,3}$, Ch. Stütz ${ }^{2}$, and W. W. Weiss ${ }^{2}$ \\ 1 Tavrian National University, Yaltinskaya 4, 330000 Simferopol, Crimea, Ukraine \\ e-mail: vad@ccssu.crimea.ua \\ ${ }^{2}$ Institute for Astronomy, University of Vienna, Türkenschanzstraße 17, 1180 Vienna, Austria \\ 3 Institute of Astronomy, Russian Academy of Science, Pyatnitskaya 48, 119017 Moscow, Russia
}

Received 7 August 2003 / Accepted 8 September 2004

\begin{abstract}
Modelling stellar atmospheres becomes increasingly demanding as more accurate observations draw a more complex picture of how real stars look like. What could be called a normal star becomes increasingly rare because of, e.g., significant deviations from the classical solar abundance pattern and clear evidence for stratification of elements in the atmospheres as well as surface inhomogeneities (spots) causing further severe deviations from "standard" atmospheres. We describe here a new code for calculating LTE plane-parallel stellar model atmospheres for early and intermediate type of stars which has been written in Compaq Fortran 95 and can be compiled for Windows and Linux/UNIX computer platforms. The code is based on modified ATLAS9 subroutines (Kurucz) and on spectrum synthesis codes written by V. Tsymbal with the main modifications of input physics concerning the block for opacity calculation. Each line contributing to opacity is taken into account for modelling the atmosphere, similar to synthetic spectrum calculations. This approach, which we call the line-by-line (LL) technique, avoids problems resulting from statistical methods (ODF, OS) and allows to calculate complex models with abundances which are not simply scaled from a standard pattern (usually the solar abundances) and which can be even depth dependent. Stratification is considered in this context as an empirical input parameter which has to be derived from observations. Due to the implemented numerical methods, mainly in the opacity calculation module, our code produces model atmospheres with modern PCs in a time comparable to that required by classical routines.
\end{abstract}

Key words. stars: atmospheres - stars: abundances - stars: chemically peculiar - stars: fundamental parameters stars: individual: CU Vir - stars: individual: HD 124224

\section{Introduction}

Modelling of stellar atmospheres (together with synthetic spectrum calculations) is one of the most important tools for investigating stellar structure and evolution and for determining their main parameters. Starting with the first simple and analytical models (Eddington 1929) the growing knowledge about physical processes in a plasma allowed to calculate more realistic models, taking into account such effects as line blanketing (Strom \& Kurucz 1966) and deviations from the local thermodynamic equilibrium (Mihalas \& Auer 1970).

One of the main problems for stellar atmosphere modelling was the huge amount of spectral lines which have to be taken into account; up to tens of millions. To save computing time, several statistical methods for the representation of line absorption have been developed. Best known are the Opacity Distribution Function method (ODF) (see Strom \& Kurucz 1966; Querci et al. 1974; Gustafsson et al. 1975; Kurucz 1979), first implemented by Labs (1951), and the Opacity Sampling (OS) method (Peytremann 1974; Sneden et al. 1976; Johnson \& Krupp 1976, Carbon 1984, Ekberg et al. 1986; Alexsander et al. 1989; etc.). Due to their statistical nature, these methods have various shortcomings discussed in, e.g., Carbon (1974), Castelli \& Kurucz (1994) and references therein.

Approximations have to be used to facilitate model atmosphere calculations. In general, the atmosphere is sliced into layers which are considered to be plan-parallel, level populations are assumed to be in local thermal equilibrium (LTE) and the chemical composition is considered to be constant throughout the entire atmosphere. For a long time the latter assumption was not disputed and the ODF method was very popular because of its modest demand for computer power. It was sufficient to calculate ODF tables for a limited number of chemical compositions (usually scaled to the solar case), temperatures, pressures, microturbulent velocities, and to use these tables when calculating model atmospheres of normal stars.

Recently more and more attention is being paid to the problem of model atmospheres with individual abundances. Moreover, in particular in the case of chemically peculiar (Ap) stars, it is necessary to take stratification of elements into account, because spectra of these stars indicate a vertical abundance distribution with sometimes steep gradients (Wade et al. 2001; Ryabchikova et al. 2002). In such a case line opacity 
calculations pose a serious problem which, for the ODF method, would require to calculate ODF tables for each layer. Already a full calculation of model atmospheres with constant, but individual abundances (including line preselection procedures and calculation of ODF tables) is very time consuming. Such an approach has been chosen by Piskunov \& Kupka (2001) for modelling CP stars with individual abundances.

The accuracy of the opacity sampling (OS) method depends directly on the number of frequency points used. Increasing this number one can expect a more accurate representation of line absorption effects and thus of the model structure. This method was chosen for ATLAs12 (Kurucz 1993) and PHOENIX (Hauschildt et al. 1997; Baron \& Hauschildt 1998, and references therein).

In this paper we present a new software package which allows to calculate plane-parallel, LTE models for early and intermediate types of stars using, what we call, the line-byline method for computing line opacity effects. The package is based on the main program blocks of ATLAS9 (continuum opacity, temperature correction, evaluation of the Saha equation) and on STARSP (Tsymbal 1996) for calculating the line absorption.

In the next Section we briefly describe the main features of the new code and the strategy for the calculations, followed by a comparison with other codes, such as ATLAS9, ATLAS12 and PHOENIX, for a standard model with $T_{\text {eff }}=10000 \mathrm{~K}$, $\log g=4.0$, and $[\mathrm{A} / \mathrm{H}]=0$. We also tested our code on the Si star CU Vir (HD 124224) and compared our results to those obtained by the advanced ATLAS9 code developed by Piskunov \& Kupka (2001).

We show that the stratification of iron plays a major role for the famous $\lambda 5200 \AA$ depression which is enhanced in the spectrum of CU Vir at the phase of light maximum.

\section{Brief description of the code}

\subsection{Line-by-line calculation}

What we call in this paper the "line-by-line" (LL) technique is used to compute the line absorption coefficient on a very fine wavelength grid including also opacities caused by other lines at both sides from the given grid point. The step size for flux integration has to be chosen small enough to provide a sufficiently accurate description of all line profiles contributing to the opacity. In other words, line-by-line is the direct method for line absorption calculation which was not yet used regularly, because of the limitations in computing resources. Obviously, there is no difference between LL and OS methods, provided the latter is based on a grid of 300 000-500000 points in the spectral range of the maximum stellar radiation. In such a case, the frequency and depth dependence of the line absorption coefficient is completely covered. Hence, when calculating LL model atmospheres we reproduce the spectrum in detail, almost in the same manner as for spectrum synthesis.

Figure 1 shows the flux calculated with ATLAS12 (top) and with the LL code (bottom) for an arbitrarily chosen wavelength region. ATLAS 12 employs an approximately $1 \AA$ step size while we use $0.1 \AA$. Obviously quite some lines are missed with the

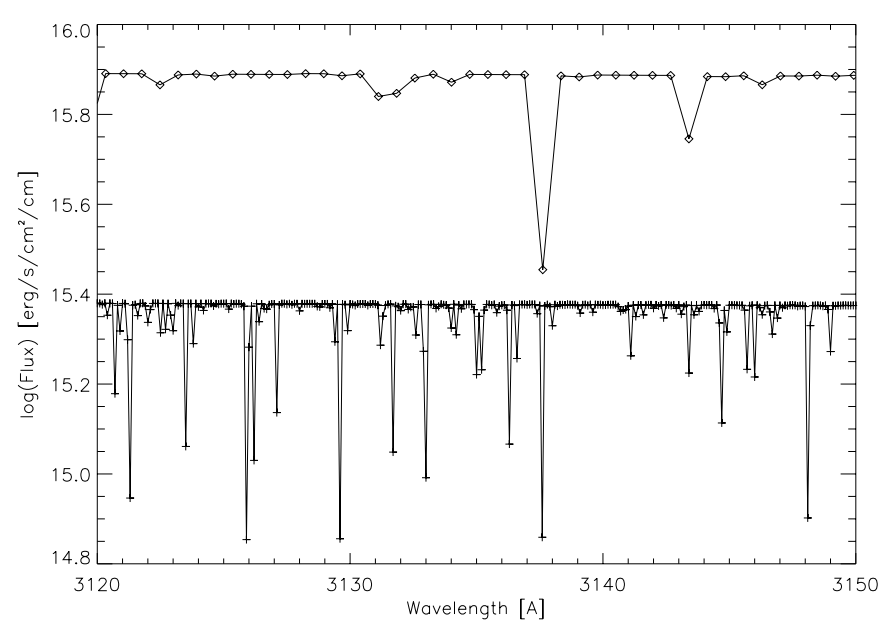

Fig. 1. Comparison between the ATLAS12 (top curve) and the line-byline (bottom curve) flux calculation. The same line list was used for both calculations. Diamonds and crosses show the wavelength points used by the two codes, respectively. Fluxes obtained by the LL calculation have been off-set by -0.5 in the logarithmic scale. Note, that OS and LL are not intended to compute a spectrum. The data points are here connected by a line only to guide the eye.

OS method. However, this deficit is not as critical as it might appear to be, because of the statistical nature of the OS method. Nevertheless, the temperature structure of a given model depends on the total flux which calls for a most realistic flux integration as is provided with the LL technique. For chemically stratified atmospheres, as is the case, e.g., for Ap stars, the contribution of the same spectral intervals to the total opacity differs in general from a uniform not-stratified atmosphere. Finally, using too large steps for the flux integration causes incorrect estimates of the total opacity in these regions. The LL method avoids completely such uncertainties.

Computing speed obviously is a crucial issue for the acceptance of our LL technique. Previous attempts to introduce the basic LL concept in routine work were realized, for example, by Morton (1965), Mihalas \& Morton (1965), Adams \& Morton (1968) who used only ninety-eight ultraviolet spectral lines and only in the region covered by Lyman series. Gustafsson et al. (1975) used this method for constructing latetype giant model atmospheres. Computing time is driven by the small wavelength steps in the spectral region which is to be integrated and by the large number of lines which have to be considered. The line absorption at a given wavelength is determined also by neighbouring lines and the number of these lines can be very large, in particular in the UV region. Thus, for each wavelength grid point also all nearby lines have to be considered when calculating the total absorption. In ATLAS12 e.g., R. Kurucz uses 200 grid points to take absorption by a single line into account. With a step size for ATLAS 12 of about $1 \AA$ this implies a spectral range comparable to a complete A-type star Balmer line!

As the wings of most atomic lines cover at most a few $\AA$, there is no need to calculate their profiles far beyond a reasonably chosen limit $\Delta \lambda$, for which the opacity contribution for a given line becomes negligible. It was found experimentally that $\Delta \lambda=2.5 \AA$ is accurate enough for the most extended 
sets of spectral lines. Thus, in our calculations we summarize opacities from all lines within a $\pm 2.5 \AA$ window centered on a given wavelength grid point. The same approach had been implemented in the STARSP code (Tsymbal 1996). Wide lines (we call "wide" those lines which still contribute to the opacity outside the $2.5 \AA$ interval), as hydrogen lines, Ca II $\mathrm{H}$ and $\mathrm{K}$ lines, etc. are treated differently.

An optimized line sorting algorithm contributes to the performance of the code. 20000 lines are extracted at once from a master list what is more than enough to compute the line opacity in $5 \AA$ intervals at any spectral region. The master list is provided from a data base, such as LOWLINES.DAT (Kurucz 1994) or VALD (Piskunov et al. 1995; Kupka et al. 1999; Ryabchikova et al. 1999) where the line density of the data base in the UV region is about 10000 lines per $\AA$. The extracted preselected lines are kept in the computer memory until new lines have to be downloaded with a fast sorting routine which replaces lines not contributing any more to the opacity in the new spectral interval.

To calculate a model with 400000 frequency points based on almost 440000 lines requires about 3-5 min per iteration and the total computing time on an Athlon $X P^{+}(2.2 \mathrm{GHz}$, 512 RAM) for a completely converged model is about $2 \mathrm{~h}$, depending on the quality of the input model parameters. For comparison, ATLAS 12 which basically uses 30000 frequency points requires approximately 1 minute per iteration (500 000 lines, Intel Celeron with $1.1 \mathrm{GHz}$ and 128 RAM) and LL calculation takes 8 min with $0.1 \AA$ and 4 min with $0.2 \AA$ wavelength steps on the same computer and with the same line list.

Variable wavelength steps (for example, small in the UV region and larger in the IR) are also a user option in our code, but in practice a constant wavelength step of $0.1 \AA$ is chosen, appropriate for the UV region. A further reduction of this step size does not change significantly the model structure. Increasing the wavelength steps towards the IR does not significantly reduce the total computing time because the atomic line density is decreasing in this region. Obviously, cool star model atmospheres with many molecular lines to be considered will be more time consuming, but such stars are not a target for our code.

One important goal of the LL method is to avoid any statistical techniques when calculating line opacities. LL and OS methods produce different results only for optically thin layers, as is illustrated in Fig. 2. We have calculated six model atmospheres with the same $T_{\text {eff }}=12700$ and $\log g=4.0$, but different numbers of frequency points. Abundances and atomic line data were the same and the integration of the radiation field parameters was always carried out between 100 and $40000 \AA$. The wavelength step was chosen to be $0.01 \AA$, $0.05 \AA$, $0.1 \AA$, $0.5 \AA$ and $1 \AA$. Finally, a model was calculated with wavelength steps similar to ATLAS12 (30000 points between 500 and $500000 \AA$ ). Models with stepsizes of up to $0.1 \AA$ A have almost the same temperature distribution and the maximum deviation does not exceed $20 \mathrm{~K}$ (Fig. 2). These differences rapidly increase for more coarse grids and in particular in the uppermost layers.

The differences between LL- and OS-based model atmospheres are not that important in the chemically homogeneous

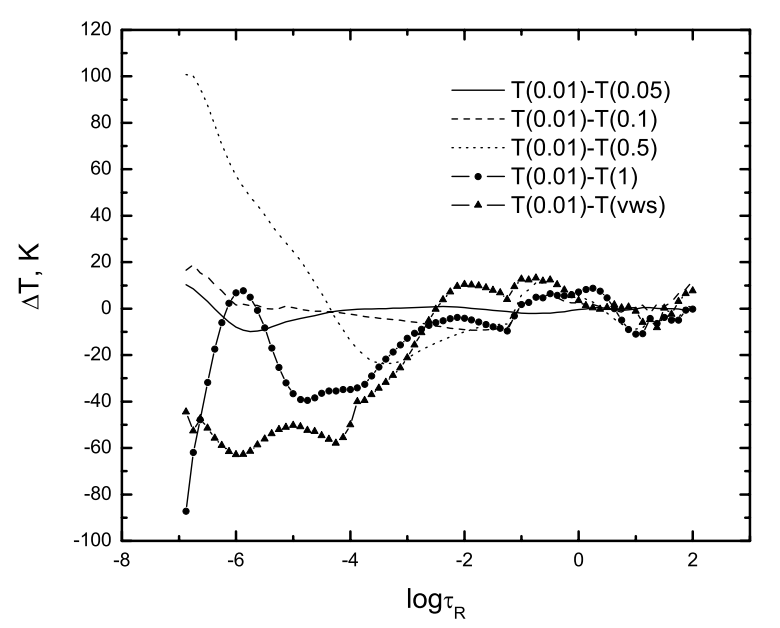

Fig. 2. Temperature differences between models calculated with different wavelength steps relative to a model computed with 0.01 A steps. The values in brackets give the wavelength steps used in Angstrom. The last model (vws) was calculated with a variable wavelength grid close to that of ATLAS12.

case, but they are critical for layers near the surface of inhomogeneous atmospheres. For example, some of the rare-earth elements in the Ap star atmosphere of $\gamma$ Equ show strong overabundance above $\tau_{R}=10^{-6}$ (see Ryabchikova et al. 2002). In this case it is indeed important to have accurate models for these layers to assure a correct determination of the abundance stratification.

We therefore see the following arguments in favour of the LL models:

- they provide a more realistic description of the stellar atmosphere structure. Frequency and depth dependency of line absorption coefficients are correctly described;

- they do not rely on a statistical method as does ODF or OS. Therefore they are free of approximations inherent to the latter techniques;

- the LL method has a higher dynamical range in opacity, resulting in a more realistic description of stellar atmospheric surface layers;

- due to the implemented numerical algorithms and in spite of the large number of frequency points used, the LL method allows to compute models with modern PC's in reasonable times.

\subsection{Continuum and hydrogen lines}

The continuum absorption coefficient calculated with a $1 \AA$ wavelength step does not change the model structure in comparison to an "exact" calculation. We therefor use the the same step size of $1 \AA$ for continuum and hydrogen line profiles except for a $\pm 50 \AA$ interval centered on the line core where the line absorption is calculated with the same small wavelength step used for the other lines. For hydrogen line profiles we are using Stark broadening tables from Lemke (1997) based on the VCS broadening theory (Vidal et al. 1973). The wide line opacities, such as for $\mathrm{Ca}$ II $\mathrm{H}$ and $\mathrm{K}$, are also calculated with the 
fine wavelength grid as is used for all other lines, but for an increased wavelength interval of $\pm 30 \AA$.

\subsection{Hydrostatic equation}

The hydrostatic equation is solved similar to ATLAS9 with Hamming's predictor-corrector method (for details see Kurucz 1970; or Heiter et al. 2002). The relevant equation is

$\mathrm{d} P_{\text {total }}=\frac{g}{\kappa} \mathrm{d} \tau$

or, in logarithmic scale

$\frac{\mathrm{d} \ln P_{\text {total }}}{\mathrm{d} \ln \tau}=\frac{g \tau}{\kappa P_{\text {total }}}$

where

$P_{\text {total }}=P_{\text {gas }}+P_{\text {rad }}+P_{\text {turb }}$

and $P_{\text {gas }}, P_{\text {rad }}$ and $P_{\text {turb }}$ are, respectively, gas, radiative and turbulent pressure. In ATLAS 9 and ATLAS 12 this equation is solved using tables of $\kappa_{\text {Ross }}$ calculated simultaneously with ODF tables for chosen pairs of $T$ and $P_{\text {gas }}$, microturbulent velocity $\xi_{\mathrm{t}}$, and abundances typically scaled to the solar case. But precalculated ODF and $\kappa_{\text {Ross }}$ tables are unhandy for models with abundances significantly different from the scaled solar values. Especially for models with chemical stratification this procedure is time consuming, because it requires an integration over a frequency interval:

$\frac{1}{\kappa_{\text {Ross }}}=\frac{\int \frac{1}{\kappa_{v}} \frac{\mathrm{d} B_{v}}{\mathrm{~d} T} \mathrm{~d} v}{\int \frac{\mathrm{d} B_{v}}{\mathrm{~d} T} \mathrm{~d} v}$

where $\kappa_{v}$ is the total absorption coefficient at the frequency $v$ and $B_{v}$ is the Planck function.

Instead of $\kappa_{\text {Ross }}$ we use the monochromatic optical depth $\tau_{5000}$ and determine the absorption coefficient at $5000 \AA$. After $T_{\text {new }}$ and $P_{\text {new }}$ were calculated they are back interpolated on the standard $\tau_{\text {Ross }}$ grid to prevent models from fluctuating along atmospheric depths. We continue working in terms of $\tau_{\text {Ross }}$.

\subsection{Convection}

Convective transport of energy in stellar atmospheres influences the

- pressure profile due to a turbulent pressure $P_{\text {turb }}$;

- thermal contribution to the energy flux in the atmosphere;

- line opacity, because turbulent velocity fluctuations cause additional Doppler broadening.

The ATLAS9 code treats convection with the classical mixing length concept (MLT, see Biermann 1948; Öpik 1950; Böhm-Vitense 1958; Castelli et al. 1997) which is based on a characteristic scale length $l$, defined as a fraction $\alpha$ of the local pressure scale height

$H_{\mathrm{p}}=\frac{P_{\mathrm{tot}}}{\rho g}=\frac{l}{\alpha}$
The examples for an inadequacy of the MLT concept are numerous. We just mention here that $\alpha$ needs to be about 0.5 to fit the Balmer line profiles for the Sun and the cool dwarfs (Fuhrmann et al. 1993), but has to be chosen between 1 and 2 to reproduce their stellar radii (Morel et al. 1994). To reproduce the whole red giant branch in HR diagrams of galactic open clusters $\alpha$ has also to be tuned (Stothers \& Chin 1995, 1997).

An alternative to MLT was introduced by Canuto \& Mazzitelli $(1991,1991)$ who tried to improve the physical description of convection while keeping computational expenses as low as for MLT. The CM convection model has been implemented in our code in the same manner as it was done for ATLAS9 by Heiter et al. (2002).

\subsection{Convergence of the models}

Constancy of the total flux and conservation of radiative equilibrium are the two convergence criteria used for our models. Both criteria are checked after each iteration and for each atmospheric depth. If the condition

$\left(H_{\mathrm{rad}}+H_{\text {conv }}\right)-\sigma T_{\mathrm{eff}}^{4}=0$

and the total energy balance

$\int\left(\alpha_{v}+\ell_{v}\right) J_{v} \mathrm{~d} v-\int\left(\alpha_{v}+\ell_{v}\right) S_{v} \mathrm{~d} v=0$

are matching accross the boundary layers with errors of less than $1 \%$, we assume the model to be converged. Here, $H_{\text {rad }}$ and $H_{\text {conv }}$ are radiative and convective fluxes, $T_{\text {eff }}$ is the effective temperature, $\alpha_{v}$ and $\ell_{v}$ are continuum and line absorption coefficients at a frequency $v, J_{v}$ and $S_{v}$ are the mean intensity and source functions, respectively. Note that the first criterion is not very sensitive to temperature variations in the optically thin layers. In this case the second criterion (radiative equilibrium) becomes more relevant which is the reason why it was introduced. If at least one of these criteria cannot be matched, either the initial model parameters were inappropriate or the model does not converge due to limitations of the implemented numerical algorithms.

\section{Comparison of different codes}

\subsection{Standard model}

To test our code and to check for possible errors we compared models $\left(T_{\text {eff }}=10000 \mathrm{~K}, \log g=4.0,[\mathrm{~A} / \mathrm{H}]=0.0\right)$ produced by three different codes: ATLAS9, ATLAS 12 and PHOENIX. First, we used LOWLINES.DAT, a line list produced by Kurucz (1994) for selecting all the lines for which $\ell_{v} / \alpha_{v} \geq 1 \%$, with $\ell_{v}$ and $\alpha_{v}$ being the line and continuum absorption coefficients, respectively. This criterion is sufficient for the chosen model and indeed, we calculated models with $10^{-4} \%, 10^{-2} \%$ and $0.5 \%$ as a preselection criterium and found temperature differences not exceeding $20-30 \mathrm{~K}$ for the surface layers. The total list of relevant lines contains after this preselection about 200000 entries. The spectral region was chosen from 500 to $30000 \AA$, that is where the star radiates most of its flux, and wavelength steps of $0.1 \AA$. 


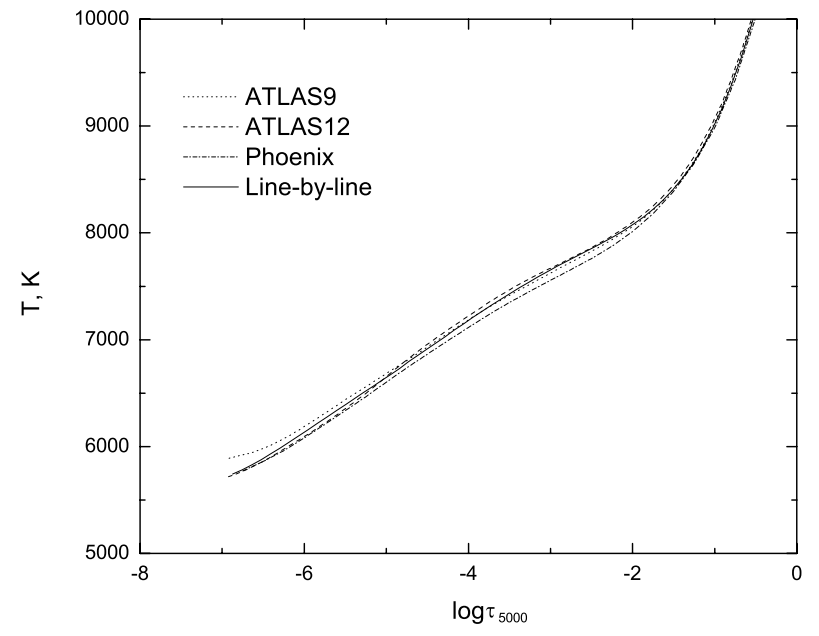

Fig. 3. Temperature distribution calculated with four codes: ATLAS9 (dotted line), ATLAS12 (dashed line), PHOENIX (dashed-dotted line) and LL (full line)

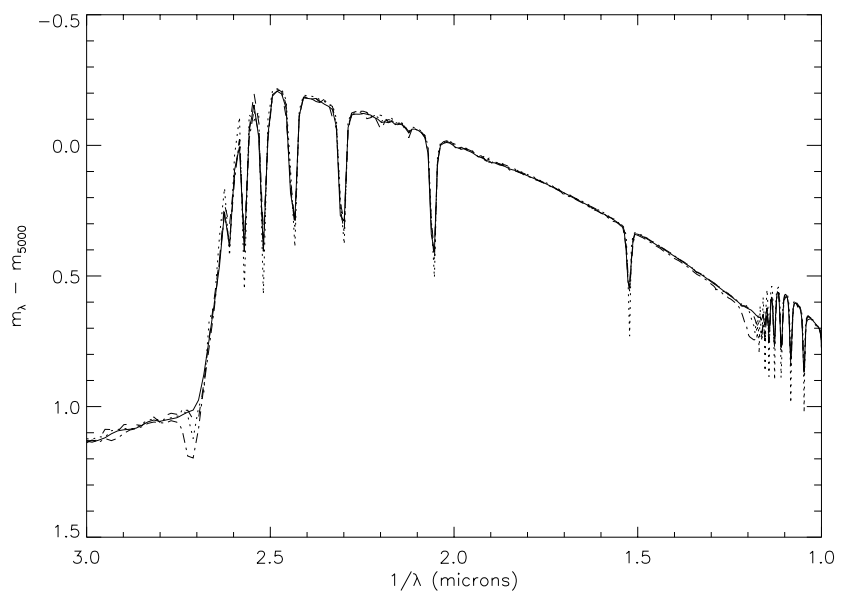

Fig. 4. Flux comparison. The symbols are the same as for Fig. 3.

Figure 3 illustrates the temperature distribution obtained by the four codes in the line forming region. Obviously, all codes provide almost the same results. ATLAS9 produces higher temperatures in the outermost surface layers, but these differences are not severe and are caused by shortcomings of the ODF method (see Castelli \& Kurucz 1994).

The energy distributions are shown in Fig. 4. Fluxes obtained by ATLAS12, PHOENIX and by LL (our code) were convolved with a Gaussian of $20 \AA F W H M$. Evidently, differences exist only near the limits of the hydrogen series where ATLAS9, ATLAS12 and PHOENIX produce flux deficits relative to LL. Most probably, this is due to the different theories used to calculate hydrogen line cross sections. As already mentioned, we implemented the VCS theory (Lemke 1997), while the other codes use the broadening theory of Griem (1960) which is not valid for the line cores. They are systematically deeper and thus cause a flux deficit. In conclusion, our LL code is in very good agreement with the other codes.

The implementation of the CM convection model was tested with ATLAS9 models of effective temperatures between $6000 \mathrm{~K}$ and $9000 \mathrm{~K}$, surface gravities ranging from 2.0

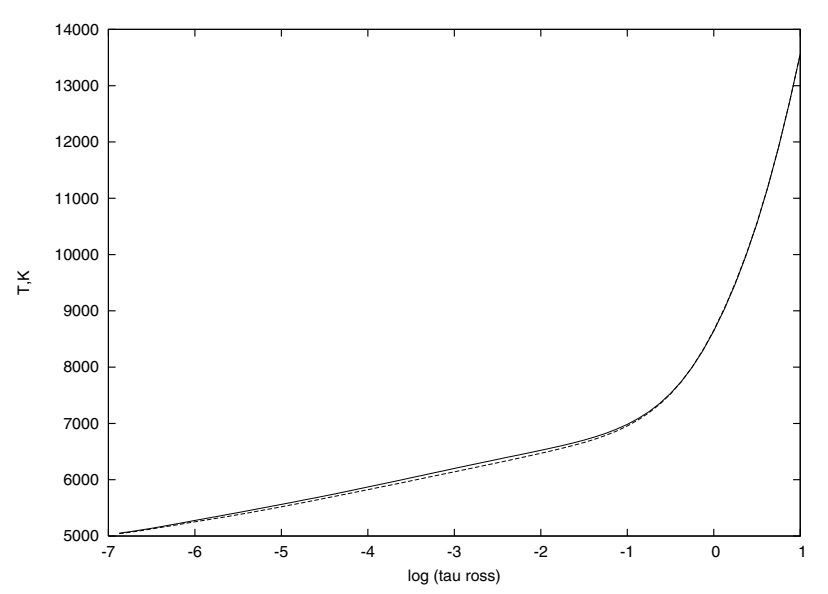

Fig. 5. CM convection treatment for LL and ODF standard models $\left(T_{\mathrm{eff}}=8000 \mathrm{~K}\right.$ and $\left.\log g=4.0,[\mathrm{~A} / \mathrm{H}]=0\right)$. Full line $-\mathrm{LL}$ calculation, dashed line - ODF method.

to 4.0, and using solar abundances. In Fig. 5 we show a model with solar abundances, $T_{\text {eff }}=8000 \mathrm{~K}$ and $\log g=4.0$. Model atmospheres are most sensitive to convection treatment for such parameters. The maximum deviation at $\log \tau_{R}=-3$ between LL and ATLAS9 models - using the same description of convection $(\mathrm{CM})$ - is less than $60 \mathrm{~K}$. In addition, we checked the models for the peculiar star $\beta \mathrm{CrB}$ and for the $\delta$ Scuti variable FG Vir and obtained similar results.

\subsection{Models with individual abundances}

Next we checked our code for stars with abundances very different from the solar case. For this purpose we chose the well studied magnetic B9p Si star CU Virginis (HD 124224) which shows strong surface abundance inhomogeneities. Variability of the Balmer line profiles was found by Ryabchikova (1972) and Weiss et al. (1976). Doppler Images show surface abundance variations of He (Hiesberger et al. 1995), Si (Goncharski et al. 1983; Hatzes 1997) and some others elements (Kuschnig et al. 1999). Temperature and gravity variations at the surface of this very rapidly rotating star are probably responsible for the observed Balmer line profile variations (Kuschnig et al. 1999).

The starting model parameters and abundances for $\mathrm{He}, \mathrm{Mg}$, $\mathrm{Si}, \mathrm{Cr}$ and $\mathrm{Fe}$ were taken from Kuschnig et al. (1999). The authors noted that the best fit for the $\mathrm{H}_{\delta}$ line can be obtained with ATLAS9 model atmospheres of same $T_{\text {eff }}$, but different gravities for different rotation phases:

\begin{tabular}{ccc}
\hline \hline Phase & $T_{\text {eff }}$ & $\log g$ \\
\hline 0.0 & $2500 \pm 200 \mathrm{~K}$ & $3.9 \pm 0.1$ \\
0.5 & $2500 \pm 200 \mathrm{~K}$ & $4.2 \pm 0.1$ \\
\hline
\end{tabular}

In the following we compare our results with those from a code developed by Piskunov \& Kupka (2001) based on ATLAS9 and using individual ODF tables to adapt for abundance changes. To model the atmosphere of CU Vir at maximum (phase 0.0) and minimum (phase 0.5) brightness we used the flux distributions (Pyper \& Adelman 1985) and $\mathrm{H}_{\delta}$ line profiles (Kuschnig et al. 1999) observed at these phases. From the latter paper we 
Table 1. Abundances which were taken differently to the solar values when modelling CU Vir. Units of the abundances are $\log \frac{N}{N_{\mathrm{H}}}$.

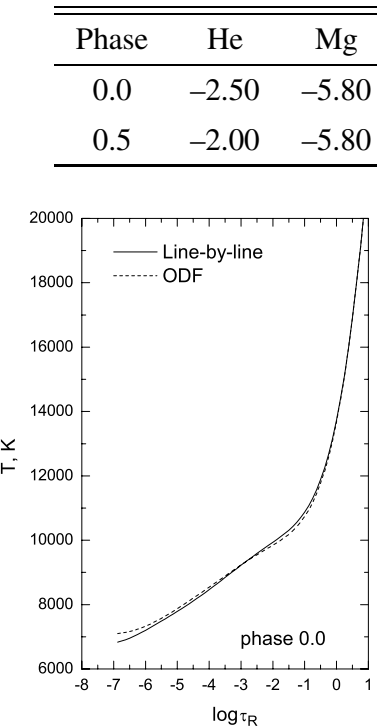

\begin{tabular}{cccc}
$\mathrm{Mg}$ & $\mathrm{Si}$ & $\mathrm{Cr}$ & $\mathrm{Fe}$ \\
-5.80 & -3.40 & -5.40 & -4.00 \\
-5.80 & -3.90 & -6.00 & -4.50 \\
\hline
\end{tabular}

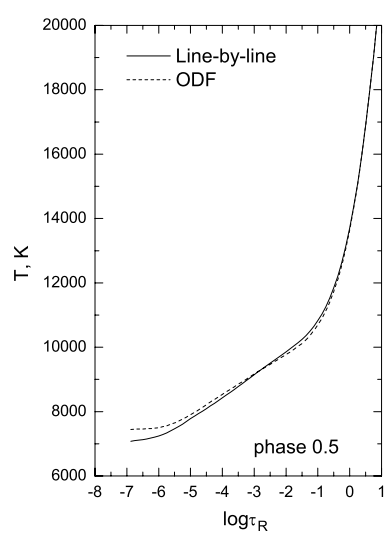

Fig. 6. Temperature distribution for CU Vir and for rotation phase 0.0 (left) and 0.5 (right). Full line - LL calculation, dashed line ODF method.

also took the average abundances (Table 1) and assumed solar abundance for all other elements.

First, we selected all lines from the Vienna Atomic Line Database (VALD) which contribute to the opacity between 500 and $40000 \AA$. This same sample was used for the ODF and LL calculations and amounted to 445000 and 333000 lines for phase 0.0 and 0.5 , respectively. For the LL calculations we used the default wavelength step of $0.1 \AA$ and achieved the best fit to the observations with the following model parameters:

\begin{tabular}{cccc}
\hline \hline Phase & $T_{\text {eff }}$ & $\log g$ & $v \sin i$ \\
\hline 0.0 & $12750 \pm 100 \mathrm{~K}$ & $4.0 \pm 0.05$ & $160 \mathrm{~km} \mathrm{~s}^{-1}$ \\
0.5 & $12750 \pm 100 \mathrm{~K}$ & $4.2 \pm 0.05$ & $160 \mathrm{~km} \mathrm{~s}^{-1}$ \\
\hline
\end{tabular}

Figure 6 shows the temperature distribution for the phases 0.0 and 0.5. The ODF method gives in both cases higher temperatures in the upper layers. At phase 0.5, e.g., this difference amounts to about $350 \mathrm{~K}$. However, this effect does not influence significantly the $\mathrm{H}_{\delta}$ profile and both codes give good agreement (Fig. 7). The flux distributions (Fig. 8) are also very similar, except for the region bluewards the Balmer jump where ODF calculations give slightly smaller fluxes probably due to an overestimate of the total absorption.

CU Vir is known to have a variable $\lambda 5200 \AA$ flux depression which is clearly stronger at phase 0.0 (Fig. 8, bottom panel, around $1 / \lambda=1.9)$. Neither LL calculation nor the ODF approach with chemically homogeneous atmospheres are able to fit this region well. We investigated stratification of those elements which significantly contribute to line opacities and deviate from solar abundance values, like $\mathrm{Si}$ and Fe. Neither the $\mathrm{H}_{\delta}$ and Si II line profiles nor the $\lambda 5200 \AA$ depression change noticeably even if the stratification of $\mathrm{Si}$ is severe. But using the

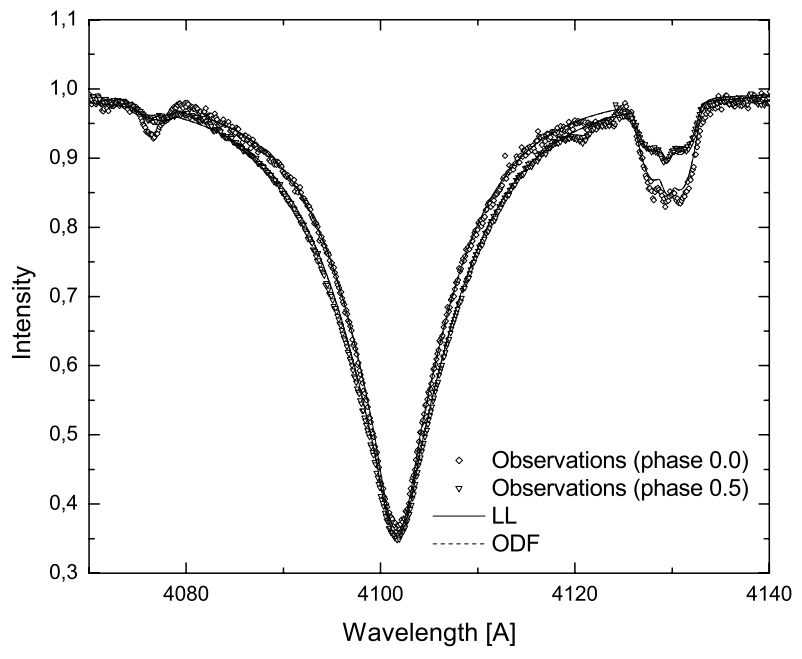

Fig. 7. Comparison between the calculated and the observed $\mathrm{H}_{\delta}$ line profiles of CU Vir for rotation phase 0.0. Full line - LL calculation, dashed line - ODF method. No difference can be seen between LL and ODF.

Fe stratification as is illustrated in Fig. 9 we were able to fit simultaneously the $\lambda 5200 \AA$ depression, the total observed flux distribution (Fig. 8, full line) and the $\mathrm{H}_{\delta}$ profile.

To investigate particularly the effects of iron on fluxes and line profiles observed at the two extreme rotation phases we computed models with homogeneous iron abundances ranging from $\log \left(\mathrm{Fe} / N_{\mathrm{H}}\right)=-3.5$ to -3.0 , characterizing the situation deeper in the atmosphere of CU Vir (Fig. 9). An increase of Fe abundance provides a better fit to the $\lambda 5200 \AA$ depression, while the overall fit to the flux becomes worse (see Fig. 10. The model with $\log g=4.5$ and $\log \left(\mathrm{Fe} / N_{\mathrm{H}}\right)=-3.0$ fits both, the $5200 \AA$ depression and reasonably well the UV region. However, with the latter parameters the $\mathrm{H}_{\delta}$ line profile cannot be fit satisfactorily. The obvious solution of the problem seems to be stratification of iron, which to handle requires a technique similar to LL.

\section{Conclusions}

With modern PCs it is possible to compute sufficiently fast stellar model atmospheres with individual and stratified abundances, using for the line absorption effects what we call the line-by-line technique. Optimized numerical techniques allow to achieve this goal without pre-computed tables and even for a dense wavelength grid (typical wavelength steps of $0.1 \AA$ ) and using several hundred thousands of atomic lines. The results for comparable models are in good agreement with those computed with ATLAS9, ATLAS12 and PHOENIX, thus providing an important test for our code. We also tested our code with stars departing significantly from solar scaled abundances, in particular with the B9p Si star CU Vir which shows strong surface abundance inhomogeneities. Overabundance and vertical stratification of iron in the atmosphere of CU Vir can explain the observed $\lambda 5200 \AA$-depression at rotational phase 0.0 , and at the same time the observed flux and the $\mathrm{H}_{\delta}$ line profile.

In conclusion, we have shown that our code allows to compute on a routinely basis model atmospheres with complex 

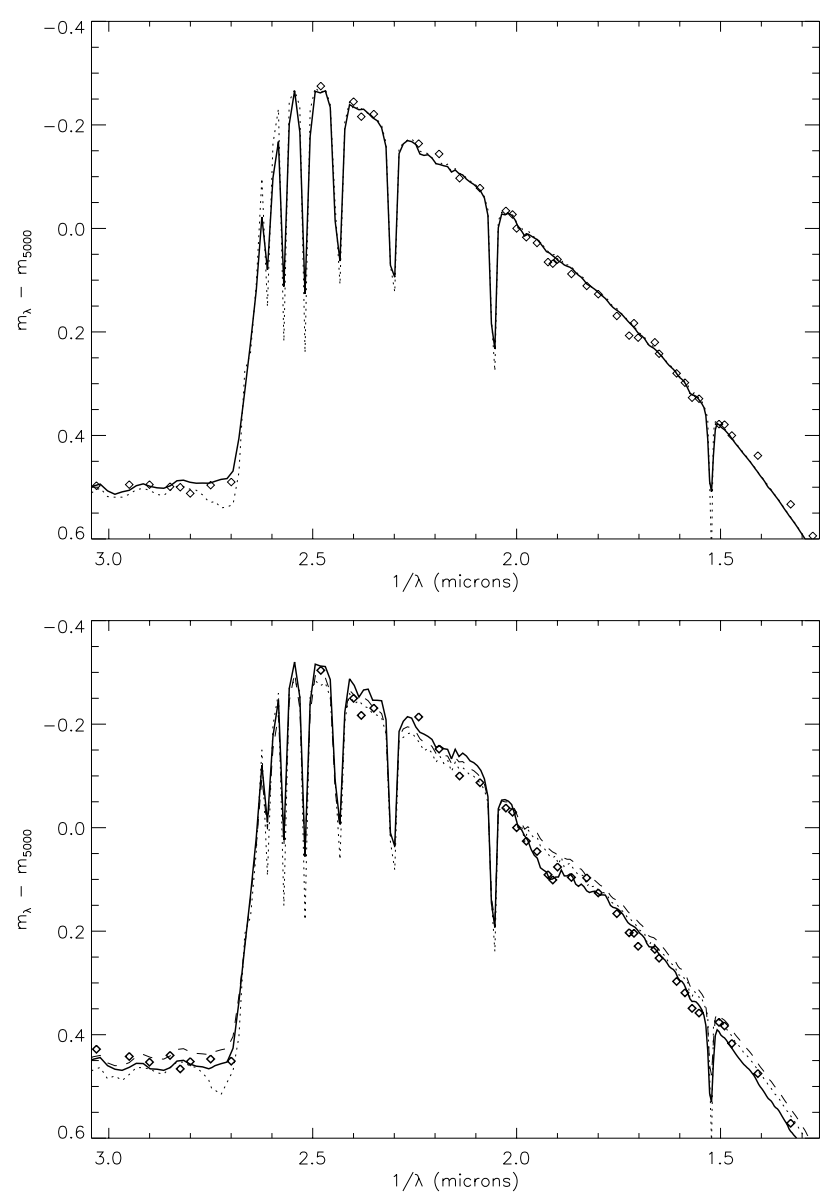

Fig. 8. The upper figure shows the energy distribution of CU Vir for phase 0.5 . Full line - LL calculations, dotted line - ODF method, diamonds - observations. The energy distribution at phase 0.0 is displayed at the bottom for the case of stratified (full line) and nonstratified (dashed line) Fe abundance. Dotted line - ODF method, diamonds - observations.

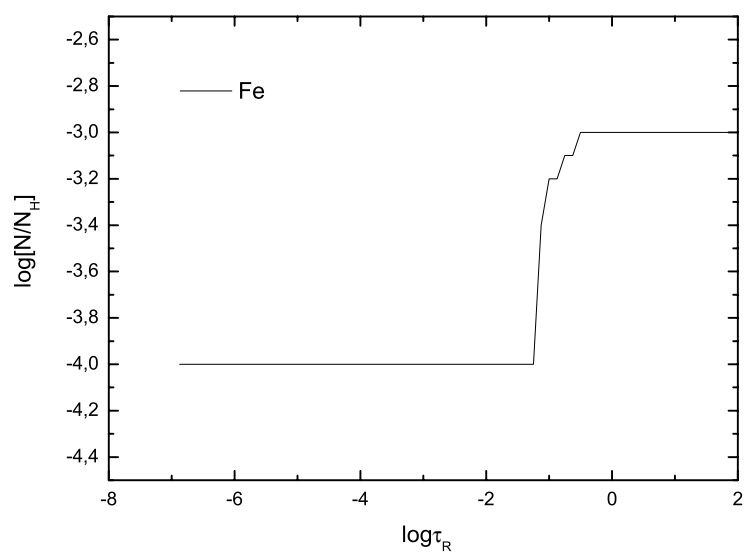

Fig. 9. The proposed stratification profile for $\mathrm{Fe}$ (dotted line) in the atmosphere of CU Vir at phase 0.0 .

properties, like any reasonable abundance pattern deviating from the solar case and which can be even changing with depth. This development provides a further step towards a consistent treatment of atmospheres of "abnormal" stars.

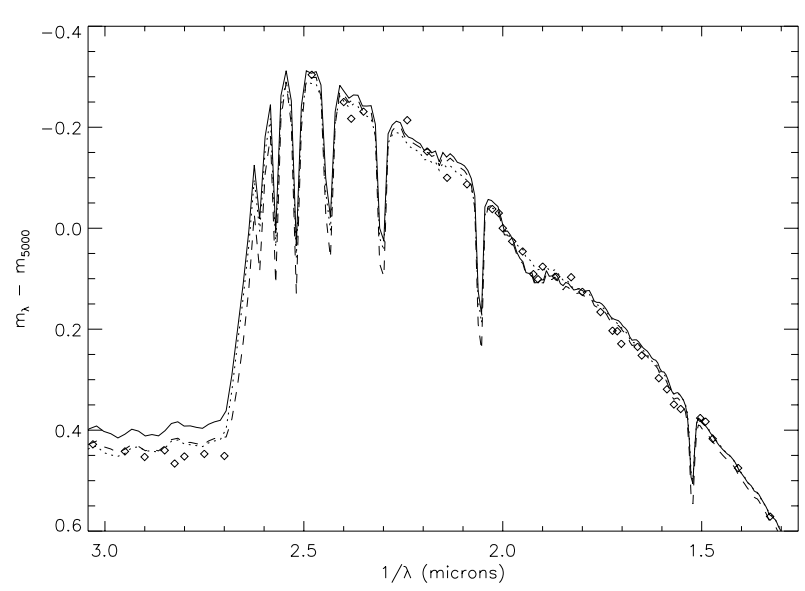

Fig. 10. Energy distributions of CU Vir for phase 0.0 (LL calculation). Full line - homogenous $\log \left(\mathrm{Fe} / N_{\mathrm{H}}\right)=-3.0$, dotted line - with $\log \left(\mathrm{Fe} / N_{\mathrm{H}}\right)=-3.5$, dashed line - with $\log \left(\mathrm{Fe} / N_{\mathrm{H}}\right)=-3.0$ and $\log g=4.5$. Diamonds - observations.

Acknowledgements. We are very much indepted to Fiorella Castelli and Francis LeBlanc for providing us with the ATLAS12 and PHOENIX models. This research was funded by the Austrian Fonds zur Förderung der wissenschaftlichen Forschung (P-14984), the $B M: B W K$ and $A S A$ (project COROT). Financial support came from the RFBR (grant 03-02-16342) and from a Leading Scientific School, grant 162.2003.02. We are also grateful to Nicolaj Piskunov, Uppsala, for his numerous useful comments and discussions.

\section{References}

Adams, T., \& Morton, D. 1968, ApJ, 152, 195

Alexsander, D., Augason, G., \& Johnson, H. 1989, ApJ, 345, 1014

Baron, E., \& Hauschildt, P. H. 1998, ApJ, 495, 370

Biermann, L. 1948, ZAp, 25, 135

Böhm-Vitense, E. 1958, ZAp, 46, 108

Canuto, V. M., \& Mazzitelli, I. 1991, ApJ, 370, 295

Canuto, V. M., \& Mazzitelli, I. 1992, ApJ, 389, 724

Carbon, D. 1974, ApJ, 187, 135

Carbon, D. 1984, in Methods in Radiative transfer, ed. W. Kalhofen (Cambrige Univ. press)

Castelli, F., \& Kurucz, R. L. 1994, A\&A, 281, 817

Castelli, F., Gratton, R., \& Kurucz, R. L. 1997, A\&A, 318, 841, erratum: 1997, A\&A, 324, 432

Eddington, A. S. 1929, MNRAS, 89, 620

Ekberg, U., Eriksson, K., \& Gustafsson, B. 1986, A\&A, 167, 304

Fuhrmann, K., Axer, M., \& Gehren, T. 1993, A\&A, 271, 451

Goncharski, A. V., Ryabchikova, T. A., Stepanov, V. V., Khokhlova, V. L., \& Yagola, A. G. 1983, Sov. Astron., 27, 49

Griem, H. R. 1960, ApJ, 132, 883

Gustafsson, B., Bell, R. A., Eriksson, K., \& Nordlund, A. 1975, A\&A, 42, 407

Hatzes, A. P. 1997, MNRAS, 288, 153

Hauschildt, P. H., Baron, E., \& Allard, F. 1997, ApJ, 483, 390

Heiter, U., Kupka, F., van't Veer-Menneret, C., Barban, C., et al. 2002, A\&A, 392, 619

Hiesberger, F., Piskunov, N. E., Bonsack, W. K., et al. 1995, A\&A, 196, 473

Johnson, H. R., \& Krupp, B. M. 1976, ApJ, 206, 201

Kupka, F., Piskunov, N. E., Ryabchikova, T. A., Stempels, H. C., \& Weiss, W. W. 1999, A\&AS, 138, 119

Kurucz, R. L. 1970, SAO Special Report, No. 308 
Kurucz, R. L. 1979, ApJS, 40, 1

Kurucz, R. L. 1993, in Peculiar versus Normal Phenomena in Atype and Related Stars, ed. M. M. Dworetsky, F. Castelli, \& R. Faraggiana, IAU Coll., 138, ASP Conf. Ser., 44, 87

Kurucz, R. L. 1994, Atomic Data for Opacity Calculations, Kurucz CD-ROM No.1

Kuschnig, R., Ryabchikova, T. A., Piskunov, N. E., Weiss, W. W., \& Gelbmann, M. J. 1999, A\&A, 348, 924

Labs, D. 1951, ZAp, 29, 199

Lemke, M. 1997, A\&AS, 122, 285

Mihalas, D., \& Auer, L. H. 1970, ApJ, 160, 1161

Mihalas, D., \& Morton, D. C. 1965, ApJ, 142, 253

Morel, P., van't Veer, C., Provost, J., et al. 1994, A\&A, 286, 91

Morton, D. C. 1965, ApJ, 141, 73

Öpik, E. J. 1950, MNRAS, 110, 559

Peytremann, E. 1974, A\&A, 33, 203

Piskunov, N., \& Kupka, F. 2001, ApJ, 547, 1040

Piskunov, N. E., Kupka, F., Ryabchikova, T. A., Weiss, W. W., \& Jeffery, C. S. 1995, A\&AS, 112, 525

Pyper, D. M., \& Adelman, S. J. 1985, A\&AS, 59, 369
Querci, F., Querci, M., \& Tsuji, T. 1974, A\&A, 31, 265

Ryabchikova, T. A. 1972, Izv. Krymck. Astrofiz. Obs., 45, 146

Ryabchikova, T. A., Piskunov, N., Stempels, H. C., Kupka, F., \& Weiss, W. W. 1999, Phys. Scr., T83, 162

Ryabchikova, T., Piskunov, N., Kochukhov, O., et al. 2002, A\&A, 384, 545

Sneden, C., Johnson, H. R., \& Krupp, B. M. 1976, ApJ, 204, 281

Stothers, R. B., \& Chin, C. 1995, ApJ, 440, 297

Stothers, R. B., \& Chin, C. 1997, ApJ, 478, L103

Strom, S. E., \& Kurucz, R. L. 1966, JQSRT, 6, 591

Tsymbal, V. V. 1996, in Model Atmospheres and Spectral Synthesis, ed. S. J. Adelman, F. Kupka, \& W. W. Weiss, ASP Conf. Ser., 108, 198

Vidal, C. R., Cooper, J., \& Smith, E. W. 1973, ApJS, 25, 37

Wade, G. A., Ryabchikova, T. A., Bagnulo, S., \& Piskunov, N. 2001, in Magnetic Fields Across the Hertzsprung-Russell Diagram, ed. G. Mathys, S. K. Solanki, \& D. T. Wickramasinghe, ASP Conf. Ser., 248, 341

Weiss, W. W., \& Albrecht, R. 1976, A\&A, 47, 423 